\title{
PICOSECOND ABSORPTION SPECTROSCOPY OF ENERGY TRANSFER PROCESSES IN POLYMETHINE DYES
}

\author{
K.-H. FELLER \\ Chemical Department, Center of Optical Spectroscopy, Friedrich-Schiller- \\ University Jena, DDR-6900 Jena, GDR \\ R. GADONAS and V. KRASAUSKAS \\ Laser Research Center, Vilnius-State-University, Vilnius, 232054, USSR
}

(Received January 5, 1989; in final form January 31, 1989)

Results are presented of time-resolved absorption investigations of energy transfer processes between a polymethine dye and an iron complex dye (dye E). For the first time the acceptor molecule transient absorption depletion generated by energy transfer from an excited donor was observed. The decay time of the acceptor deactivation $(\approx 140 \mathrm{ps})$ in the presence of the energy donor agrees very well with the $S_{1}$-state lifetime of the energy donor.

KEY WORDS: Energy transfer, picosecond absorption spectroscopy, polymethine, electron transfer.

\section{INTRODUCTION}

The phenomenon of intermolecular excitation energy transfer has been the subject of continuous research ${ }^{1,2,3}$ due to its importance in various fields in nature and technology. In the photosynthesis the excitation energy of the light harvesting complex is transferred to the reaction center, whereby an additional transfer of the optical energy to another spectral region takes place. In laser dye mixtures energy transfer can extend the lasing range, increase the output efficiency, the stability of operation and can influence the temporal characteristics of the output pulses of dye lasers.

Fórster energy transfer processes are very efficient if the fluorescence spectrum of a possible energy donor overlaps with the absorption spectrum of the energy acceptor. The values of the critical energy transfer radius $R_{0}$ are mainly determined by this overlap. $R_{0}$ is the radius at which $50 \%$ of the excited molecules are quenched by Fórster energy transfer. ${ }^{4}$

It is given by

$$
R_{0}^{6}=\frac{9000 K^{2} \phi \ln 10}{128 \pi^{5} n^{4} N} \int_{0}^{\infty} \frac{f_{\mathrm{D}}(\tilde{v}) \varepsilon_{A}(\tilde{v})}{\tilde{v}^{4}} \mathrm{~d} \tilde{v}
$$


The meaning of the symbols are

$f_{\mathrm{D}}(\tilde{v})$ - the spectral distribution of donor fluorescence normalized to unity (in units of quanta on a wavenumber scale)

$\varepsilon_{\mathrm{A}}(\tilde{v})$ - the molar extinction coefficient

$K^{2}$ - the spatial distribution function of the excited molecules (in case of rotation of the molecules $K^{2}=2 / 3$ )

and

$N$-Avogadro-number

$\phi$-the fluorescence quantum yield

Deviations from the normal Fórster energy transfer law have been found in cases of inhomgeneous distribution ${ }^{2,3}$ of acceptor molecules around the donor molecule. The reasons for such inhomogeneous distribution can be various including electrostatic interaction in the case of oppositely charged donor and acceptor molecules. In the case of appropriate oxidation and reduction potentials the excitation energy quenching process could involve a very fast electron exchange mechanism in the primary step of the deactivation. ${ }^{5}$

It was recently shown by Feller et al $^{6}$ that the fluorescence of cationic cyanine dyes could be very efficiently quenched by various anionic dyes. In this case the rate parameters depend stronger on the interaction strength between the cationic donor and the anionic acceptor than on the overlap between the donor fluorescence and the acceptor absorption. A detailed investigation of the energy transfer process, which

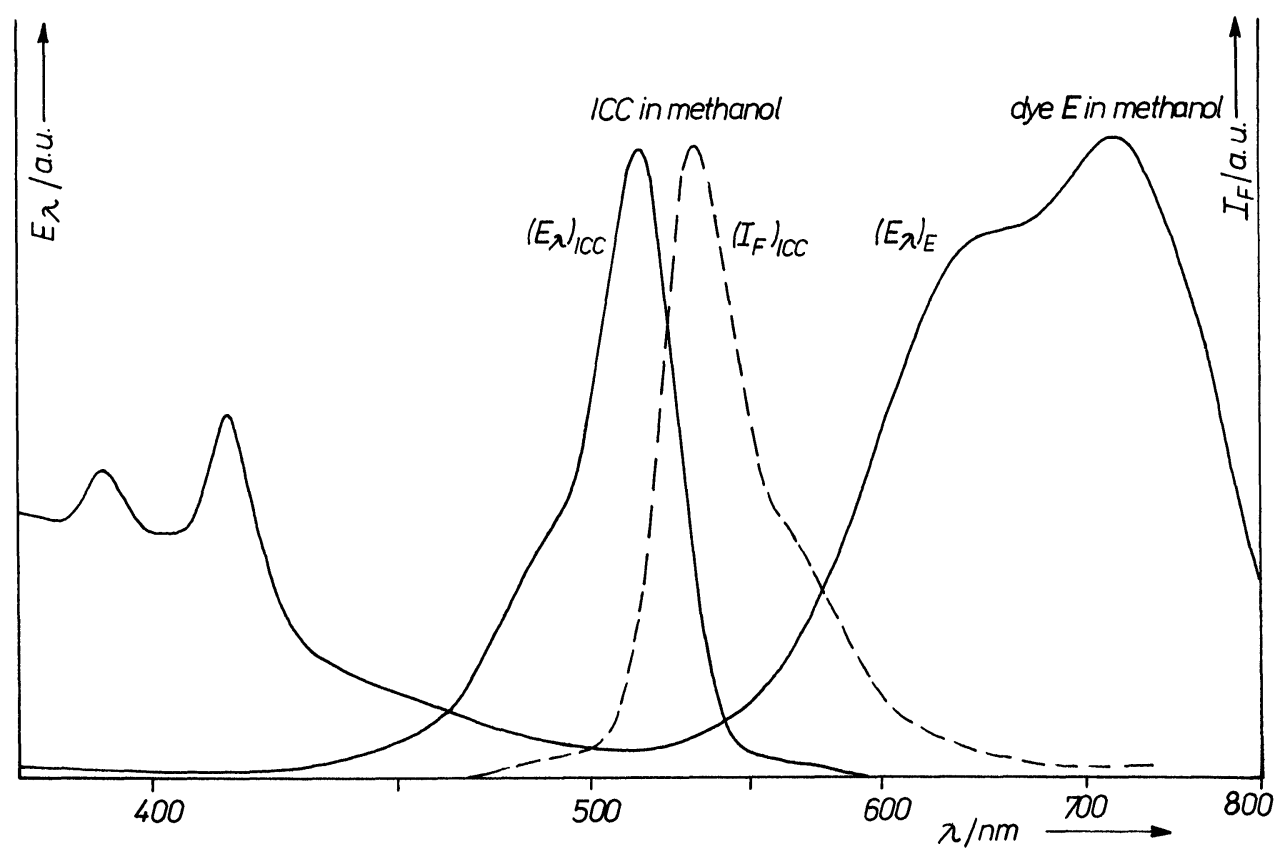

Figure 1 Absorption and fluorescence spectra of ICC and absorption spectrum of dye E in methanolic solution. 


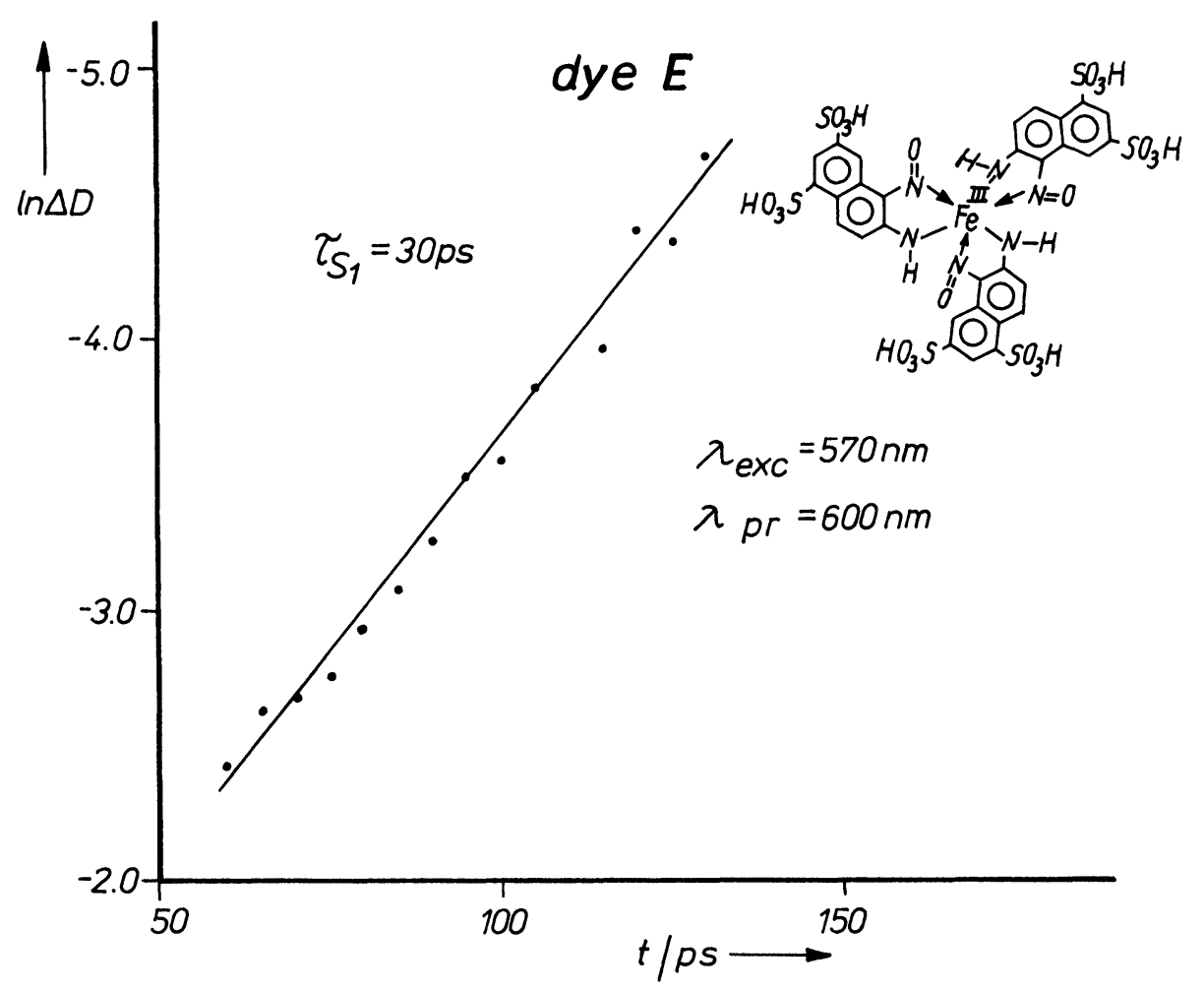

Figure 2 Ground state recovery kinetics of dye E.

takes into account electron transfer as a possible primary step, is therefore of great interest. For our experiments we have chosen an iron complex dye as energy acceptor (dye E in Figure 1, for structure see Figure 2) because of its low optical density in the absorption region of the energy donor dye ICC.

\section{EXPERIMENTAL}

The energy donor dye 1,1'-dimethyl-3,3'-di-p-N-phenylacetoaniline-6,6'-dicyanobenzimidacarbocyanine iodide (abbr.: ICC) as well as the quenching dye $\mathrm{E}$ were purified several times by column chromatography and by recrystallization. The absorption and fluorescence spectra of ICC together with the absorption spectrum of dye $\mathrm{E}$ are shown in Figure 1; the structure of dye $\mathrm{E}$ is shown in Figure 2. Dye $\mathrm{E}$ does not show any detectable fluorescence at room temperature. The used carbocyanine dye which was recently investigated in detail ${ }^{7}$ does not aggregate neither in alcoholic nor in water solution because of steric hindrance. The triplet yield as well as the photoisomerization quantum yields are less than $1 \%{ }^{8}$

The experiments were made with a pump-and-probe pulse spectrometer which has been described in detail elsewhere. ${ }^{9}$ The samples were excited in the absorption 
band of the investigated dye ( $500 \mathrm{~nm}$ in the case of ICC, $570 \mathrm{~nm}$ in the case of dye E) and the absorption spectra at different time delays as well as the kinetics in the absorption recovery region (see Figure $2-4$ ) have been probed by $30 \mathrm{ps}, 50 \mu \mathrm{J}$ probe pulses.

No evidence was found for excitation energy dependent lifetime components or spectral changes.

The fitting procedure of the lifetime components to the experimental curves were done with the help of the ALAU-program package. ${ }^{10}$

\section{RESULTS AND DISCUSSION}

The ground state recovery time of dye E was estimated from the decay curve to be 30 ps (Figure 2).

The Forster energy transfer radius was calculated from the decay curve of the ground state recovery experiments of ICC with an acceptor concentration of $c_{\text {dyeE }}$ $=5 \cdot 10^{-3} \mathrm{~mol} / \mathrm{l}$ (see Figure 3 ) by plotting the logarithm of the signal intensity against the square root of $t$ (see Figure 4). The time evolution of deactivation decay curve in the case of Forster energy transfer is

$$
I_{D}=I_{D}(0) \exp \left[-t / \tau_{s}-2 \gamma\left(t / \tau_{s}\right)^{1 / 2}\right]
$$

where the excited state lifetime $\tau_{s}$ is the lifetime in the absence of energy transfer,

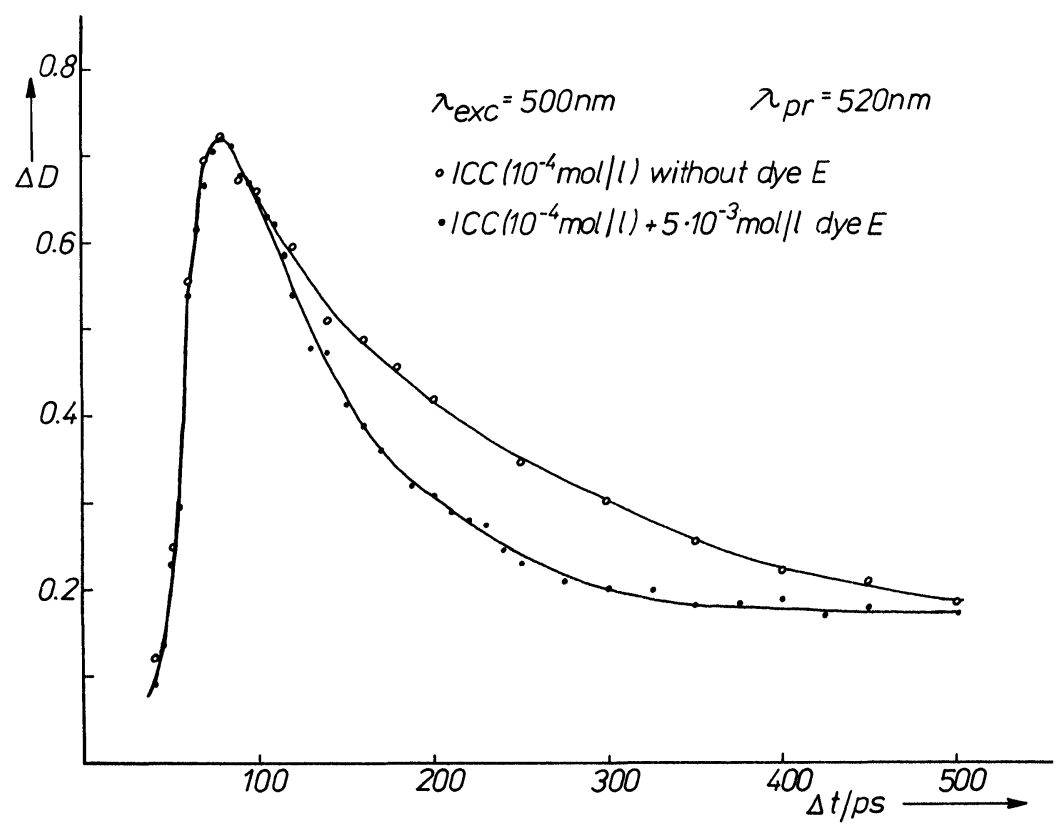

Figure 3 Ground state recovery kinetics of ICC in a methanolic ICC $\left(10^{-4} \mathrm{~mol} / \mathrm{l}\right)$ solution without dye E and with $5 \cdot 10^{-3} \mathrm{~mol} / \mathrm{l}$ dye $\mathrm{E}$. 


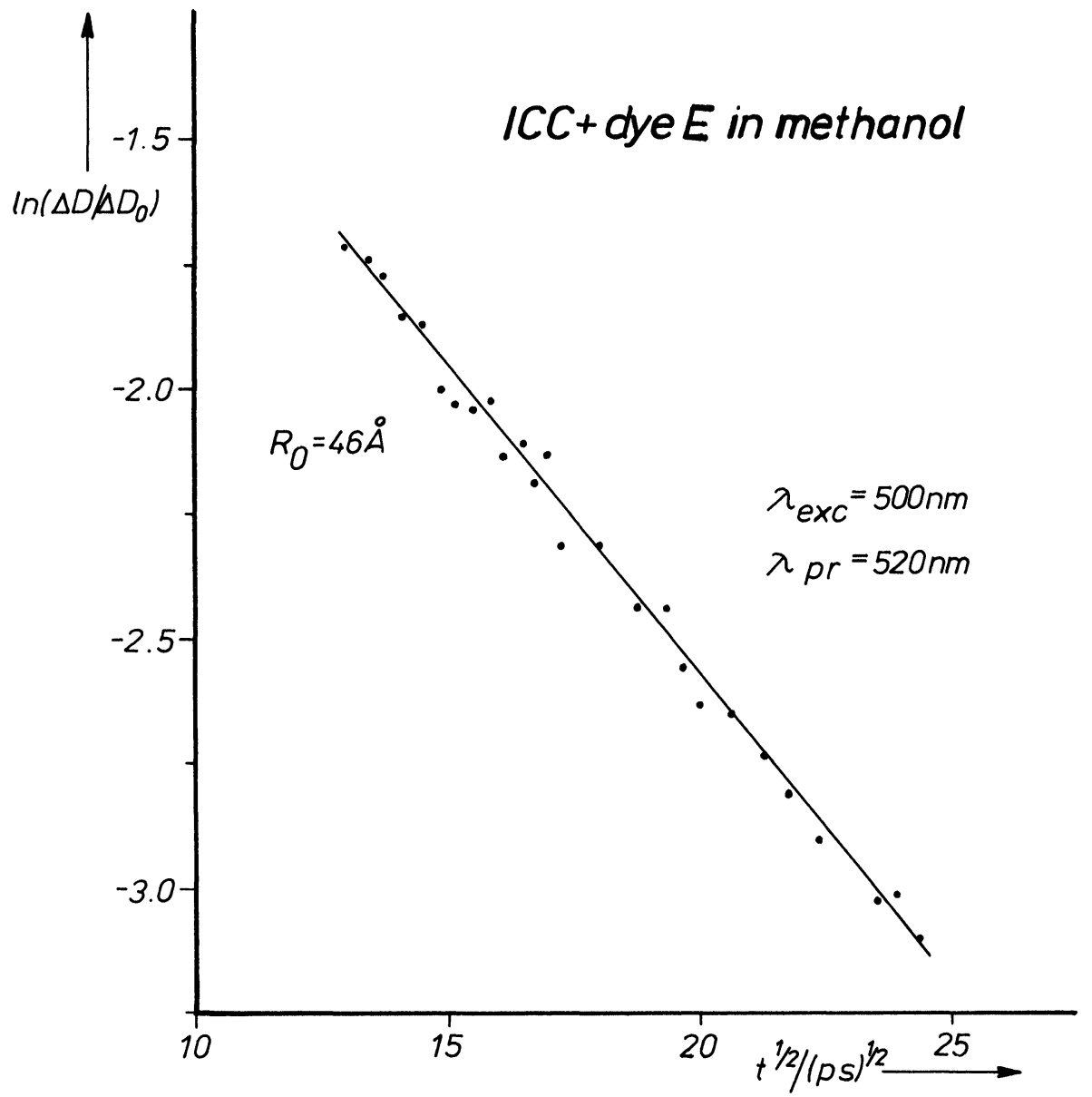

Figure 4 Semilogarithmic plot of the ground state recovery kinetics of ICC in a ICC $\left(10^{-4} \mathrm{~mol} / \mathrm{l}\right)-\mathrm{dye} \mathrm{E}$ $\left(5 \cdot 10^{-3} \mathrm{~mol} / \mathrm{l}\right)$ methanolic solution vs. square root of time.

$\gamma=c_{A} / c_{0} . c_{A}$ is the acceptor concentration and $c_{0}$ is the critical concentration given by

$$
c_{0}=\frac{3000}{2 \pi^{2 / 3} N R_{0}{ }^{3}}
$$

A graph of $\log \left[\Delta D(t) / \Delta D_{0}(t)\right]$ against $T^{1 / 2}$ where $\Delta D_{0}(t)$ is the unquenched optical density difference with $\Delta D_{\mathrm{o}}(t)=A_{0}(0)$ exp $\left(-t / \tau_{s}\right)$ gives a linear dependence of gradient $-2 \gamma / 2.303\left(\tau_{s}\right)^{1 / 2}$. Such a plot is shown in Figure 4. The energy transfer radius calculated in that way amounts to $46 \AA$ whereas the Fórster energy transfer radius calculated from the overlap integral amounts to $40 \AA$ which is a very reasonable agreement. The bleaching of the time dependent absorption spectrum of an energy acceptor was detected for the first time by excitation in the absorption band of the donor (ICC, $\lambda_{\text {exc }}=490 \mathrm{~nm}$ ) (see Figure 5). In comparison to the 


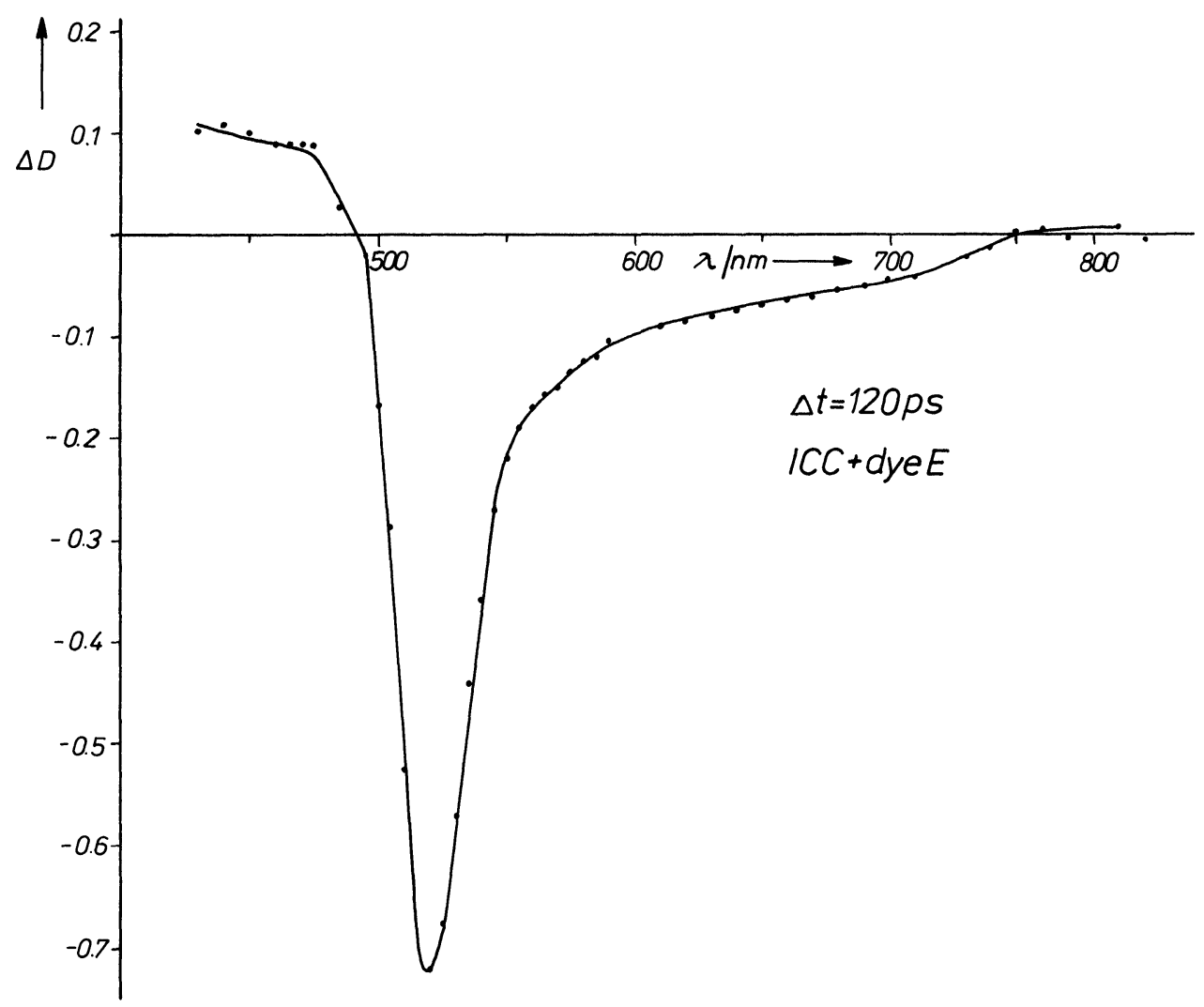

Figure 5 Bleaching curve of the transient absorption spectrum of dye $\mathrm{E}$ by excitation in the absorption band of $\operatorname{ICC}\left(\lambda_{\text {exc }}=490 \mathrm{~nm}\right)$.

transient absorption spectrum of the unquenched ICC (see Figure 2 in $^{11}$ ) the build-up of a transient absorption between $650-750 \mathrm{~nm}$ is clearly seen in Figure 5 . This transient absorption is connected with the excitation and deactivation of the dye E molecules excited via energy transfer.

In the case of very fast nonradiative deactivation of excited acceptor molecules the rise of the acceptor excited state population via energy transfer from the excited donor is a convolution of donor $S_{1}$-state decay kinetics and acceptor excitation kinetics. So in our case, where

$$
\tau_{s_{1}}(\mathrm{ICC}, 165 \mathrm{ps})>\tau_{s_{1}}(\text { dye E, } 30 \mathrm{ps}),
$$

the risetime of the transient absorption spectrum of dye $E$ is not represented by the rise time of the acceptor dye $E$ alone. To understand the decay kinetics of the excited dye $\mathrm{E}$ the following limiting cases should be taken into consideration. First case:

$$
\tau_{\text {donor }} \gg \tau_{\text {acceptor }} ; \quad 1 / k_{\text {transfer }} \leqslant \tau_{\text {donor }}
$$


In this case

Second case:

$$
c_{E}{ }^{*} \text { follows } c_{\text {donor }}{ }^{*} \text {. }
$$

In this case

$$
\tau_{\text {donor }} \ll \tau_{\text {acceptor }}
$$

$$
c_{E}^{*} \approx c_{E}^{*} \text { (directly excited). }
$$

The experimental findings are clearly in favour of the first alternative. So the decay kinetics are more represented by the excitation time behaviour of dye $E$ than by its deactivation time dependence. This gives us the approach to calculate the energy transfer time behaviour from the decay kinetics of dye $\mathrm{E}$.

We calculated from the decay of dye $E$ by excitation in the absorption band of ICC a decay time of $140 \pm 20$ ps.

$\mathrm{In}^{6}$ the involvement of a very fast electron transfer and back transfer as possible deactivation process was taken into account as explanation of the dependence of the rate constants of fluorescence quenching on the ionic interaction between donor and acceptor molecules in inhomogeneously distributed cation-anion systems as depicted in the following scheme

$$
\mathbf{D}^{+}+\mathbf{A}^{-} \stackrel{h v}{\longrightarrow} \mathbf{D}^{+*} \ldots \mathbf{A}^{-} \longrightarrow \mathbf{D} \ldots \mathbf{A} \cdot \longrightarrow \mathbf{D}^{+} \ldots \mathbf{A}^{-*} \longrightarrow \mathbf{D}^{+}+\mathbf{A}^{-}
$$

Such a behaviour would be connected with a delayed build-up of the $S_{1}$-state population of the energy acceptor, which was not observed in our experiments. As the $S_{1}$-state lifetime of ICC at this acceptor concentrations is appr. $130 \mathrm{ps}$, this results shows that the deactivation of ICC is directly connected with the excitation of the energy acceptor. This result excludes the involvement of the above mentioned very fast electron transfer mechanism as explanation of the high efficiency of the investigated quenching process. To support this result spectral changes in the dye neutral radical absorption region ${ }^{12}$ have been tried to detect. Within the experimental detection limit (time resolution in the $S_{1}-S_{n}$ absorption region of appr. 50 ps. limited sensitivity due to overlap between the $S_{1}-S_{n}$ absorption and the radical spectrum) no electron transfer was detected.

\section{References}

1. M. C. Adams, D. J. Bradley, W. Sibbett and J. R. Taylor, J. Mol. Struct. 61, 5 (1980).

2. M. Kaschke and K. Vogler, Chem. Phys. 102, 229 (1986).

3. M. Kaschke and K. Vogler, Laser Chem. 8, 19 (1988).

4. I. B. Berlman, Energy Transfer Parameters of Aromatic Compounds (New York, London; Academic Press, 1973).

5. V. Brúckner, K.-H. Feller and U.-W. Grummt, Applications of Time-Resolved Optical Spectroscopy (Elsevier Science Publishers, Netherlands, 1989).

6. K.-H. Feller, D. Fassler, P. Hampe, K. Berndt, E. Klose and P. Schwarz, Z. phys. Chemie (Leipzig) 266, 2 (1985).

7. D. Fassler and K.-H. Feller, J. Mol. Struct. 173, 377 (1988).

8. K.-H. Feller, R. Gadonas and V. Krasauskas, unpublished results.

9. R. Gadonas, R. Danielius and A. Piskarskas, Kvantovaya Elektron. 8, 669 (1981).

10. H. Schútz, E. Stutter, K. Geller and I. Petri, studia biophysica 104, 23 (1984).

11. S. Rentsch, R. Danielius and R. Gadonas, J. Signalaufz.-Mater. 12, 319 (1984).

12. K.-H. Feller, D. Fassler, R. Gadonas, V. Krasauskas, A. Pelakauskas and A. Piskarskas, J. Inf. Rec. Mat. 17(5/6) (1989). 